\title{
Successful Conservative Treatment of Spontaneous Perforated Duodenal Diverticulum: A Case Report
}

\author{
Su Hyung Park, Jung Gil Park ${ }^{1}$ \\ Department of Internal Medicine, CHA Bundang Medical Center, CHA University, Bundang, Department of Internal Medicine, CHA Gumi \\ Medical Center, CHA University ${ }^{1}$, Gumi, Korea
}

\begin{abstract}
Duodenal diverticula are common disease entities occurring in up to $25 \%$ of the healthy population. Duodenal diverticular perforation is a rare but fatal complication. Although the main treatment for duodenal diverticular perforation is surgery, conservative treatment can be an option for selected patients. We present a case of a 71-year-old woman with a perforated duodenal diverticulum successfully managed with conservative treatment with antibiotics and percutaneous drainage of abscesses. (Korean J Helicobacter Up Gastrointest Res 2016;16:34-37)
\end{abstract}

Key Words: Duodenum; Diverticulum; Perforation; Nonsurgical treatment

\section{INTRODUCTION}

In the alimentary tract, the duodenum is the second most common organ, after the colon, for the development of diverticula. ${ }^{1}$ The prevalence of duodenal diverticula in the general healthy population can reach up to $25 \%$, with most diverticula located in the second portion of the duodenum and within $2 \mathrm{~cm}$ of the ampulla of Vater. ${ }^{1,2}$ Rarely, duodenal diverticula can cause complications, such as obstruction of the common bile duct, pancreatitis, perforation, ulceration, and bleeding. Of these, duodenal diverticular perforation is the most severe complication, with a mortality of up to $30 \%$, and may be caused by diverticulitis, ulcerations, and diagnostic procedures such as endoscopy. ${ }^{3}$ Though surgery is the main treatment option, conservative treatment could be considered, depending on the patient's clinical status. Here, we describe a case of perforated duodenal diverticulum treated successfully with antibiotics and percutaneous drainage of retroperitoneal abscesses.

Received: July 11, 2015 Accepted: December 18, 2015

Corresponding author: Jung Gil Park

Division of Gastroenterology and Hepatology, Department of Internal Medicine, CHA Gumi Medical Center, CHA University, 12 Sinsi-ro 10-gil, Gumi 39295, Korea

Tel: +82-54-450-9797, Fax: +82-54-450-9546, E-mail: gsnrs@naver.com

\section{CASE REPORT}

A 71-year-old woman with no significant medical history was admitted to CHA Gumi Medical Center for evaluation of vague epigastric discomfort and poor oral intake for three days. A physical examination revealed mild right upper quadrant tenderness without muscular guarding. Upon admission, her blood pressure was normotensive and she was afebrile. The white blood cell count was 11,600 cells $/ \mathrm{mm}^{3}$ and the CRP concentration, $5.70 \mathrm{mg} / \mathrm{dL}$. Further laboratory tests found an ALT level of 57 IU/L; AST, 75 IU/L; ALP, 162 IU/L; BUN, 19.3 mg/dL; creatinine, $0.58 \mathrm{mg} / \mathrm{dL}$; and were negative for viral hepatitis serum markers. Plain abdomen radiography showed mild small bowel ileus without intra-abdominal free air. Subsequent multiphase abdominal CT revealed a $3 \mathrm{~cm}$ diverticulum and enhanced luminal wall thickening in the second portion of the duodenum with large amounts of retroperitoneal air and fluid collection (Fig. 1A). We diagnosed a duodenal diverticular perforation and retroperitoneal abscess. After insertion of a nasogastric (NG) tube, the patient was empirically treated with antibiotics. Due to refusal of surgery and the non-septic condition of the patient, we performed percutaneous drainage of the abscesses (Fig. 2). Ten days after admission, the patient showed a favorable clinical course and the NG tube was removed. An upper gastrointestinal series was performed

Copyright $\odot 2016$ Korean College of Helicobacter and Upper Gastrointestinal Research

() The Korean Journal of Helicobacter and Upper Gastrointestinal Research is an Open-Access Journal. All articles are distributed under the terms of the Creative Commons Attribution Non-Commercial License (http://creativecommons.org/licenses/by-nc/4.0) which permits unrestricted non-commercial use, distribution, and reproduction in any medium, provided the original work is properly cited. 

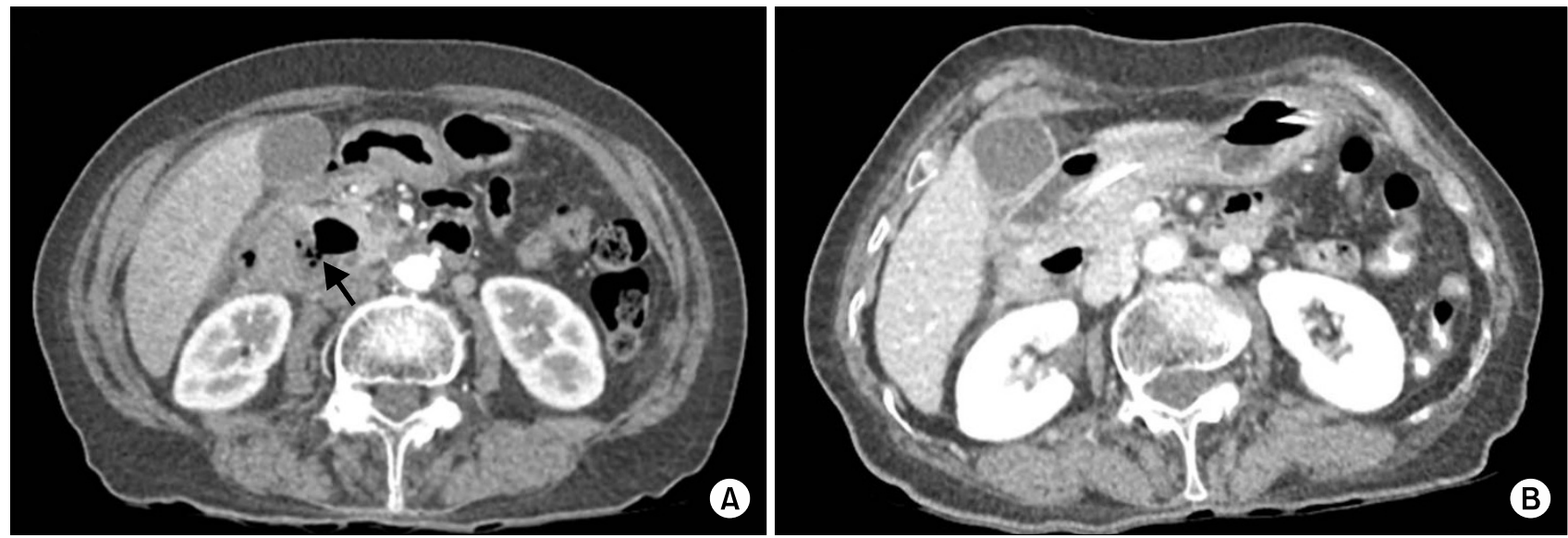

Fig. 1. Abdominal CT showing a perforated duodenal diverticulum with free air (A; arrow) at initial diagnosis and disappearance of free air and abscesses (B) after 12 days of percutaneous drainage and antibiotics.

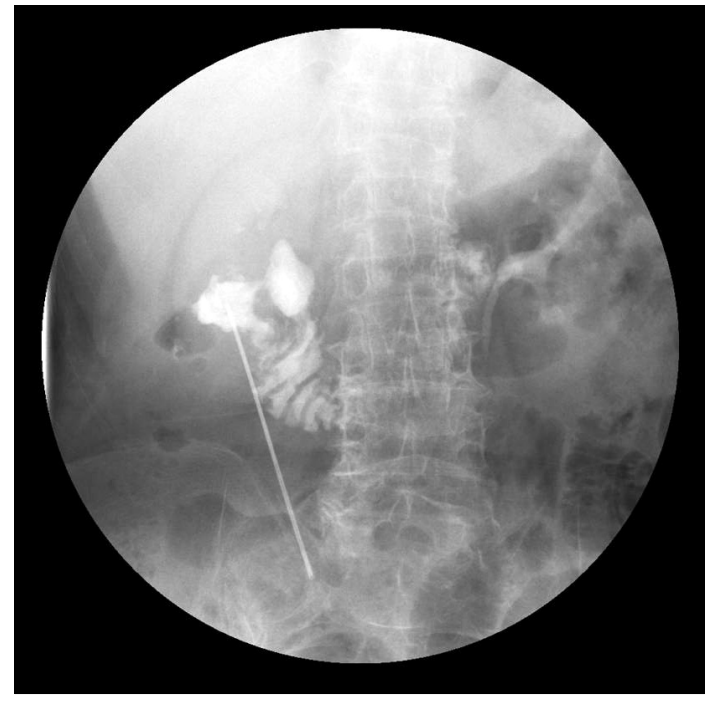

Fig. 2. Fluoroscopic image showing contrast medium leakage through the perforated diverticulum in the second portion of the duodenum during insertion of a percutaneous drainage catheter.

with gastrografin (Bayer HealthCare Pharmaceuticals, Berlin, Germany), revealing a large diverticulum without leakage of contrast medium in the second portion of the duodenum. A follow-up abdominal CT showed a decrease in size of the abscess in the anterior pararenal space and anterior side of the duodenum (Fig. 1B). After NG tube removal, the patient began a soft diet with oral antibiotics. Four weeks after discharge, an upper gastrointestinal endoscopy (TJF-240; Olympus, Tokyo, Japan) showed a suspicious erythematous lesion in the deep por-

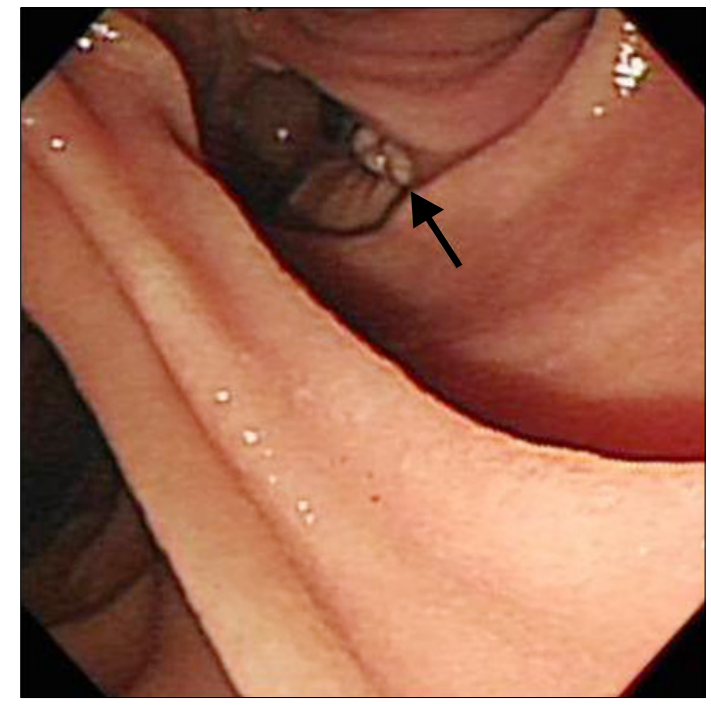

Fig. 3. Endoscopic image showing suspected granular tissue (arrow) of a healed perforated duodenal diverticulum.

tion of the diverticulum (Fig. 3). Follow-up over six months did not show any evidence of recurrence.

\section{DISCUSSION}

Duodenal diverticula usually arise from recanalization through a weak area in the duodenal lumen during gestation. ${ }^{4}$ Most duodenal diverticula are asymptomatic before complications develop. ${ }^{5}$ However, the vulnerable, thin walls of diverticula have rarely been reported to 
cause perforation. ${ }^{6}$ Duodenal diverticular perforation, first observed by Bassett in 1907 during an autopsy, is rare but highly fatal, associated with high mortality. ${ }^{3,7}$ The most common cause of duodenal diverticular perforation is diverticulitis, followed by enterolithiasis, iatrogenic, trauma, and foreign bodies. ${ }^{7}$ Because of its vague and unspecific clinical presentation, early diagnosis of perforated duodenal diverticula is difficult without imaging. ${ }^{8}$ Though there are no typical clinical features, the most common clinical symptom is right upper quadrant abdominal or epigastric pain with nausea and vomiting, mimicking acute cholecystitis, peptic ulcer disease, and pancreatitis. ${ }^{2,7,9} \mathrm{How}^{-}$ ever, chronic mild abdominal discomfort with malaise and anorexia are sometimes observed. ${ }^{10}$ Because most of the duodenal diverticular perforation is open to the retroperitoneal cavity, there may not be peritoneal signs. ${ }^{8}$ In most cases, an abdominal CT scan is regarded as the most useful diagnostic modality, as plain abdominal radiography does not show intra-abdominal free air. ${ }^{11-13}$ Typical radiological findings are extraluminal collection of free air and fluid, fat inflammation, and a thickened duodenal wall with or without diverticula. ${ }^{10,11}$ Traditional management for perforated duodenal diverticula includes a transduodenal diverticulectomy with single- or double-layer duodenal closure. $^{5,7,8}$ In addition, gastric diversion or decompression, such as gastrojejunostomy or tube duodenostomy, may be required if there is an inflamed or friable duodenal wall. ${ }^{9}$ However, the incidence of complications after surgical treatment is relatively high, around 36 $41 \%{ }^{7,9}$ Common surgical complications are duodenal fistula, suture line leaks, sepsis, residual abscesses, biliary obstruction, and pancreatitis. ${ }^{9}$ Conservative management of duodenal diverticular perforation was first reported by Shakckleton in 1963. ${ }^{7}$ In patients with mild symptoms and hemodynamic stability, conservative management, including bowel rest, intravenous hydration, broad-spectrum antibiotics, or NG tube decompression with or without percutaneous drainage of abscesses can be good treatment options. ${ }^{7,9}$ Thorson et al. ${ }^{7}$ has reviewed a total of 39 case reports and literatures producing 61 cases of perforated duodenal diverticular reported from 1989 to 2011. Fourteen patients of the 61 cases were successfully treated without surgical method and only one complica- tion was reported which developed to intra-abdominal abscess treated by percutaneous drainage successfully. They also have found that the complication rate in patients undergoing surgical treatment was higher than in the non-surgical group. In particular, mortality in the surgical group was $6 \%$, while there were no deaths in the non-surgical group. ${ }^{7}$ Conservative management of perforated duodenal diverticulum can be an initial treatment option for patients with advanced age or patients with serious cormobidities. However, if a patient's condition deteriorates or shows peritoneal signs with unstable hemodynamic status, surgical intervention should be performed. In this case, although a relatively large intra-abdominal abscess was observed in the initial abdominal CT scan, laboratory results and clinical status of the patient were stable. The case presented here suggests that clinical and laboratory findings are more important than radiological findings in determining non-surgical treatment.

In conclusion, although surgery is the mainstay of treatment for a perforated duodenal diverticulum, nonsurgical treatment may be considered for selected, clinically stable patients. This strategy could be a particularly acceptable option in elderly patients with significant comorbidities.

\section{REFERENCES}

1. Mahajan SK, Kashyap R, Chandel UK, Mokta J, Minhas SS. Duodenal diverticulum: review of literature. Indian J Surg 2004; 66:140-145.

2. Schnueriger B, Vorburger SA, Banz VM, Schoepfer AM, Candinas D. Diagnosis and management of the symptomatic duodenal diverticulum: a case series and a short review of the literature. J Gastrointest Surg 2008;12:1571-1576.

3. Özdemir Y, Güleç B, Sücüllü I, Filiz AI. A rare cause of acute abdomen: perforation of duodenal diverticulum containing ectopic pancreatic tissue. Balkan Med J 2011;28:335-337.

4. Perdikakis E, Chryssou EG, Karantanas A. Diagnosis of periampullary duodenal diverticula: the value of new imaging techniques. Ann Gastroenterol 2011;24:192-199.

5. Gonzalez LR, Pérez MMI, Iglesias PE, et al. Conservative management of perforated duodenal diverticulitis. World J Colorectal Surg 2013;3:12.

6. Ames JT, Federle MP, Pealer KM. Perforated duodenal diverticulum: clinical and imaging findings in eight patients. Abdom Imaging 2009;34:135-139.

7. Thorson CM, Paz Ruiz PS, Roeder RA, Sleeman D, Casillas VJ. 
The perforated duodenal diverticulum. Arch Surg 2012;147: 81-88.

8. Martinez-Cecilia D, Arjona-Sanchez A, Gomez-Alvarez M, et al. Conservative management of perforated duodenal diverticulum: a case report and review of the literature. World J Gastroenterol 2008;14:1949-1951.

9. Miller G, Mueller C, Yim D, et al. Perforated duodenal diverticulitis: a report of three cases. Dig Surg 2005;22:198-202.

10. Sakurai Y, Miura H, Matsubara T, Imazu H, Hasegawa S, Ochiai M. Perforated duodenal diverticulum successfully diagnosed preoperatively with abdominal CT scan associated with upper gastrointestinal series. J Gastroenterol 2004;39:379-383.

11. Bergman S, Koumanis J, Stein LA, Barkun JS, Paraskevas S. Duodenal diverticulum with retroperitoneal perforation. Can J Surg 2005;48:332.

12. Duarte B, Nagy KK, Cintron J. Perforated duodenal diverticulum. Br J Surg 1992;79:877-881.

13. Glazer GM, Buy JN, Moss AA, Goldberg HI, Federle MP. CT detection of duodenal perforation. AJR Am J Roentgenol 1981; 137:333-336. 